Влияние олокизумаба на исходы, оцениваемые пациентом с ревматоидным артритом: результаты двойного слепого рандомизированного плацебоконтролируемого многоцентрового исследования III фазы (CREDO 1)

\author{
Е.Л. Насонов ${ }^{1,2}$, Т.А. Лисицына', Е.В. Зонова ${ }^{3}$, С.М. Кузькина ${ }^{4}$
}

\begin{abstract}
${ }^{1}$ ФГБНУ «Научноисследовательский институт ревматологии им. В.А. Насоновой» 115522, Российская Федерация, Москва, Каширское шоссе, 34а 2ФГАОУ ВО «Первый Московский государственный медицинский университет имени И.М. Сеченова» Минздрава России (Сеченовский Университет) 119991, Российская Федерация, Москва, ул. Трубецкая, 8, стр. 2 ${ }^{3}$ ФГБОУ ВО «Новосибирский государственный медицинский университет» Минздрава России 630091, Российская Федерация, Новосибирск, Красный проспект, 52 ${ }^{4} \mathrm{AO}$ «Р-Фарм» 119421, Российская Федерация, Москва, Ленинский проспект, 1116
\end{abstract}

\begin{abstract}
Введение. Олокизумаб - новый ингибитор интерлейкина-6 (ИЛ-6), продемонстрировавший хорошую эффективность и безопасность для лечения взрослых пациентов с ревматоидным артритом средней и высокой степени активности в комбинации с метотрексатом при недостаточной эффективности монотерапии метотрексатом.

Цель исследования - оценить эффективность олокизумаба в отношении исходов, оцениваемых самим пациентом (patient's reported outcomes, PROs) на основании результатов, полученных в исследовании III фазы CREDO 1.

Материал и методы. В исследование были включены 428 пациентов с ревматоидным артритом, получающие препарат подкожно, которые были рандомизированы в 3 группы: 1-я группа - получавшие олокизумаб в дозе 64 мг каждые 2 недели ( $n=143)$; 2-я группа - получавшие олокизумаб в дозе 64 мг каждые 4 недели $(n=142)$; 3-я группа - получавшие плацебо каждые 2 недели $(n=143)$. PROs включали: функциональную недостаточность по индексу HAQ-DI; общую оценку активности заболевания пациентом (PtGA) по BAШ; боль по BAШ; усталость по шкале FACIT-F, качество жизни по опроснику EQ-5D, физическому и психическому компонентам шкалы SF-36. Эффективность олокизумаба оценивалась по динамике средних значений PROs и доле пациентов, сообщивших об улучшении по сравнению с исходным уровнем $\geqslant$ минимального клинически значимого различия (minimum clinically important differences, MCID) для каждого PROs к 12-й и 24-й неделям наблюдения.
\end{abstract}

Результаты. Закончили исследование 396 пациентов из 428 включенных в него. При включении в исследование пациенты разных терапевтических групп не отличались между собой по социально-демографическим показателям, длительности, активности заболевания, а также по показателям PROs. Терапия OK3 вне зависимости от режима дозирования препарата привела к значительному улучшению всех показателей, оцениваемых самими пациентами (PROs), по сравнению с плацебо через 12 недель, и это улучшение продолжилось до 24-й недели терапии. Доля пациентов, достигших и превысивших MCID через 12 и 24 недели наблюдения, была статистически значимо больше в обеих группах олокизумаба по сравнению с плацебо для общей оценки активности заболевания пациентом (PtGA), боли по ВАШ, HAQ-DI, FACIT-F. Число больных, достигших или превысивших MCID по физическому компоненту шкалы SF-36 на 12-й неделе, было статистически значимо больше в обеих группах олокизумаба, а на 24-й неделе - только во 2-й группе - по сравнению с плацебо. Психический компонент SF-36 улучшился у значимо большего процента пациентов из 2-й группы по сравнению с пациентами, получавшими плацебо, на 12-й и 24-й неделях, в то время как показатели пациентов 1-й группы по улучшению психического компонента SF-36 статистически значимо не отличались от таковых у пациентов 3-й группы.

Выводы. Терапия олокизумабом пациентов с ревматоидным артритом средней и высокой степени активности ассоциируется со значимым улучшением всех исходов, оцениваемых самим пациентом (PROs). He отмечено существенной разницы между режимами дозирования олокизумаба.

Ключевые слова: ревматоидный артрит, ингибиторы ИЛ-6, олокизумаб, CREDO 1, исходы, оцениваемые пациентом

Для цитирования: Насонов ЕЛ, Лисицына ТА, Зонова ЕВ, Кузькина СМ. Влияние олокизумаба на исходы, оцениваемые пациентом с ревматоидным артритом: результаты двойного слепого рандомизированного плацебо-контролируемого многоцентрового исследования III фазы (CREDO 1). Научно-практическая ревматология. 2021;59(1):62-69.

\section{THE EFFECT OF OLOKIZUMAB ON RHEUMATOID ARTHRITIS PATIENT'S REPORTED OUTCOMES: RESULTS OF A DOUBLE-BLIND RANDOMIZED PLACEBO-CONTROLLED MULTICENTER PHASE III TRIAL (CREDO 1)}

\author{
Evgeny L. Nasonov ${ }^{1,2}$, Tatiana A. Lisitsyna ${ }^{1}$, Elena V. Zonova ${ }^{3}$, Sofia M. Kuzkina ${ }^{4}$
}

Background. Olokizumab is a new interleukin-6 (IL-6) inhibitor that has demonstrated good efficacy and safety for the treatment of adult patients with moderate to high-grade activity of rheumatoid arthritis in combination with methotrexate with insufficient efficacy of methotrexate monotherapy.

Aim of the study was to evaluate the effectiveness of olokizumab in relation to the patient's reported outcomes (PROs) based on the results obtained in the CREDO 1 phase III study.

Material and methods. The study included 428 patients with rheumatoid arthritis who were randomized into 3 groups: group 1 - patients who received $64 \mathrm{mg}$ of olokizumab subcutaneously every 2 weeks (q2w) $(n=143)$; group 2 - 
patients who received $64 \mathrm{mg}$ of olokizumab every 4 weeks (q4w) $(n=142)$; group 3 - patients who had placebo q2w $(n=143)$. PROs included: Health Assessment Questionnaire-Disability Index (HAQ-DI); Patient Global Assessment of Disease Activity (PtGA-VAS); Subject's Assessment of Pain (VAS); fatigue according to the FACIT-F scale; quality of life according to the EQ-5D questionnaire; physical and mental components of the SF-36 scale. The effectiveness of olokizumab was evaluated by the dynamics of the average PROs values and the proportion of patients who reported improvement compared to the baseline level of $\geqslant$ minimum clinically important differences (MCID) for each PROs by weeks 12 and 24 of follow-up.

Results. 396 patients out of 428 included completed the study. When included in the study, patients of different therapeutic groups did not differ in socio-demographic indicators, duration, activity of rheumatoid arthritis, as well as in PROs indicators. Olokizumab therapy, regardless of the dosage regimen of the drug, resulted in a significant improvement in all PROs compared to placebo after 12 weeks, and this improvement sustained until 24 weeks of therapy. The proportion of patients who reached and exceeded MCID at weeks 12 and 24 of follow-up was statistically significantly higher in both olokizumab groups compared to placebo for PtGA, VAS pain, HAQ-DI, FACIT-F. The number of patients who reached or exceeded the MCID on the physical component of the SF-36 scale at week 12 was significantly higher in both olokizumab groups, and at week 24 only in the group 2 compared to the placebo group. The mental component of SF-36 improved in a significantly higher percentage of patients in the group 2 compared to placebo group at weeks 12 and 24, while the group 1 did not significantly differ from placebo group in improving the mental component of SF-36.

Conclusions. Olokizumab therapy in patients with moderate to high-grade activity of rheumatoid arthritis is associated with a significant improvement in all PROs. There was no significant difference between the dosage regimens of olokizumab.

Keywords: rheumatoid arthritis, IL-6 inhibitors, olokizumab, CREDO 1, patient's reported outcomes

For citation: Nasonov EL, Lisitsyna TA, Zonova EV, Kuzkina SM. The effect of olokizumab on rheumatoid arthritis patient's reported outcomes: results of a double-blind randomized placebo-controlled multicenter phase III trial (CREDO 1). Nauchcno-Practicheskaya Revmatologia = Rheumatology Science and Practice. 2021;59(1): 62-69 (in Russ.).

doi: $10.47360 / 1995-4484-2021-62-69$

Ревматоидный артрит (РА) - иммуновоспалительное (аутоиммунное) ревматическое заболевание неизвестной этиологии, характеризующееся хроническим эрозивным артритом и системным поражением внутренних органов, приводящее к ранней инвалидности и сокращению продолжительности жизни пациентов [1]. У пациентов с РА часто наблюдается снижение качества жизни в отношении как физического функционирования, так и эмоционального состояния из-за боли, скованности, усталости, инвалидизации и депрессии [3, 4]. Согласно современным рекомендациям, для всесторонней оценки прогрессирования заболевания и эффективности лечения РА следует оценивать исходы, сообщаемые самим пациентом (patient's reported outcomes, PROs) [5-8]. Примечательно, что, по мнению пациентов с РА, показатели, характеризующие качество их жизни, более важны в оценке эффективности терапии, чем стандартные показатели клинической активности заболевания, определяемые врачом [9].

В настоящее время для лечения РА разработан широкий спектр инновационных генно-инженерных биологических препаратов (ГИБП) [10], среди которых особый интерес представляют моноклональные антитела против рецептора (Р) интерлейкина-6 (ИЛ-6) или самого ИЛ-6 [11-13]. Олокизумаб (OK3) - гуманизированное моноклональное антитело (IgG4 каппа), специфически нейтрализующее ИЛ-6, фармакокинетические характеристики, эффективность и безопасность которого при РА были доказаны в рандомизированных контролируемых исследованиях (РКИ) фазы І и в двух клинических исследованиях фазы II [14-17]. В РКИ фазы III CREDO 1 (NCT02760368) изучалась эффективность подкожной формы препарата OK3 в дозе 64 мг каждые 2 недели (к2н) и каждые 4 недели (к4н) у пациентов со среднетяжелым и тяжелым РА, недостаточно контролируемым терапией метотрексатом (МТ). OK3 в обеих дозировках оказался значительно эффективнее плацебо в отношении снижения активности РА вне зависимости от пола, индекса массы тела, уровня ревматоидного фактора (РФ) и/или антител к циклическому цитруллинированному пептиду (АЦЦП), длительности РА [18, 19]. В мае 2020 г. ОК3 был зарегистрирован Министерством здравоохранения РФ для лечения взрослых пациентов с РА средней и высокой степени активности в комбинации с МТ при недостаточной эффективности монотерапии МТ. Данные РКИ CREDO 1, касающиеся эффективности ОК3 в отношении компонентов PROs (боль, усталость, функциональные способности, качество жизни), представлены в настоящей публикации.

\section{Материал и методы}

В исследование были включены 428 пациентов с РА из России, Беларуси и Болгарии, рандомизированных в 3 группы, получавших препарат подкожно: 1-я группа - ОК3 в дозе 64 мг к2н (ОК3 64 мг к2н) $(n=143)$; 2-я группа - ОК3 в дозе 64 мг к4н (ОК3 64 мг к4н) $(n=142)$; 3-я группа - плацебо к2н $(n=143)$. Длительность исследования составила 24 недели терапии и 20 недель последующего наблюдения/перехода в открытую фазу. Закончили исследование 396 больных.

Критериями включения в исследование были: возраст старше 18 лет; достоверный диагноз РА длительностью $\geqslant 12$ недель
${ }^{1}$ V.A. Nasonova Research Institute of Rheumatology 115522, Russian

Federation, Moscow, Kashirskoye Highway, $34 \mathrm{~A}$

${ }^{2}$ I.M. Sechenov First Moscow State Medical University of the Ministry of Health Care of Russian Federation (Sechenov University) 119991, Russian Federation, Moscow, Trubetskaya str., 8, building 2

${ }^{3}$ Novosibirsk State Medical University 630091, Russian Federation, Novosibirsk, Krasny avenue, 52 ${ }^{4} \mathrm{AO}$ «R-Pharm»

119421, Russian Federation, Moscow, Leninskiy avenue, 111B

Контакты: Лисицына Татьяна Андреевна, e-mail: talisitsyna@ rambler.ru

Contacts: Tatiana Lisitsyna, e-mail: talisitsyna@rambler.ru

Поступила 23.07.2020 Принята 26.01.2021 
до скрининга; недостаточный эффект терапии МТ (в течение $\geqslant 12$ недель до скрининга); умеренная или высокая активность РА ( $\geqslant 6$ болезненных и припухших суставов из 68); уровень С-реактивного белка (СРБ) выше нормальных значений. Активность РА оценивалась по индексу DAS28-СРБ и CDAI [20]. Исходы, которые оценивали сами пациенты (PROs) включали: функциональную недостаточность по индексу HAQ-DI (от 0 до 3 баллов) $[5,6]$; общую оценку активности заболевания пациентом (ООАЗП; Patient's Global Assessment of Disease Activity, PtGA) (от 0 до 100 мм) по визуально-аналоговой шкале (ВАШ) [5]; боль по ВАШ (от 0 до 100 мм) [5]; усталость по шкале FACIT Fatigue (Functional Assessment of Chronic Illness Therapy) (от 0 до 52 баллов) [21]; качество жизни по опроснику EQ-5D (European Quality of Life- Five-Dimension Questionnaire) [22] и физическому и психическому компонентам шкалы SF-36, включающим физическое функционирование, влияние физического состояния на повседневную ролевую деятельность (работу, выполнение повседневных обязанностей), интенсивность боли, общее состояние здоровья, жизненную активность, социальное функционирование, ролевое функционирование, обусловленное эмоциями и психическое здоровье [23]. Для каждого компонента шкалы SF-36 счет кодировали, суммировали и трансформировали по шкале от 0 (худшее состояние) до 100 (наилучшее состояние) [23].

Эффективность терапии ОКЗ оценивалась по динамике критериев Американской коллегии ревматологов (American College of Rheumatology (ACR) 20/50/70) [24], индекса DAS28-СРБ [25], клинического индекса активности болезни (CDAI) [26] через 12 и 24 недели, а также средних значений PROs и числа пациентов, сообщивших об улучшении по сравнению с исходным уровнем $\geqslant$ минимального клинически значимого различия (minimum clinically important differences, MCID) [27] для каждого PROs к 12-й и 24-й неделям наблюдения. Средние баллы доменов SF-36 определялись в исходном состоянии и через 12 и 24 недели и сравнивались с соответствующими возрасту и полу нормативными значениями для исследуемой популяции [28]. MCID по сравнению с исходными значениями считали следующим образом: для HAQ-DI снижение $\geqslant 0,22$ балла [29]; для ООАЗП (PtGA) по ВАШ и боли по ВАШ - уменьшение $\geqslant 10$ мм [22]; для усталости по FACIT-F - увеличение $\geqslant 4$ баллов [21]; для качества жизни по физическому и психическому компонентам шкалы SF-36 - увеличение $\geqslant 2,5$ балла [30].

\section{Статистический анализ}

Статистический анализ выполнялся для популяции пациентов, которым было назначено лечение (intention-to-treat, ITT), и включал всех рандомизированных пациентов. Участники исследования были проанализированы в соответствии с группой лечения, в которую они были рандомизированы. Из всех конечных точек, представленных в данной статье, HAQ-DI была единственной вторичной конечной точкой эффективности, остальные конечные точки эффективности относились к поисковому (исследовательскому) анализу. Данная непрерывная конечная точка анализировалась с использованием модели ANCOVA, скорректированной на исходное значение (различия считались статистически значимыми при $p<0,0125$ для сравнения каждого из режимов введения
ОКЗ с плацебо). Для всех исследовательских конечных точек эффективности была представлена описательная статистика. Для ООАЗП (ВАШ), выраженности боли (ВАШ), HAQ-DI, SF-36 и FACIT-F оценивалось абсолютное и относительное количество пациентов, сообщающих как минимум об улучшении (MCID), достигшем минимальной клинически значимой разницы по сравнению с исходным. Для всех непрерывных исследовательских конечных точек эффективности средние значения рассчитывались методом наименьших квадратов (значения LSM из модели ANCOVA) по группам лечения. Для определения разницы средних значений, рассчитанных методом наименьших квадратов (LSM), между каждым из протоколов OK3 и плацебо были предусмотрены 97,5\% доверительные интервалы (ДИ). Статистическую значимость разности пропорций между группами ОКЗ и плацебо определяли при помощи точечных оценок и 97,5\% ДИ методом Ньюкомба - Уилсона. Различия считались статистически значимыми, если 97,5\% ДИ не включали ноль. Для статистической обработки результатов использовалась программа Statistical Analysis System ${ }^{\circledR}$, версия не ниже 9.4 (SAS Institute, Inc., США).

\section{Результаты}

Исследование закончили 396 из 428 включенных в него пациентов. При включении в исследование пациенты разных групп не отличались между собой по социально-демографическим показателям, длительности и активности РА (табл. 1). Активность РА у пациентов всех групп была высокой (DAS28-СРБ >5,1; число болезненных (ЧБС) и припухших (ЧПС) суставов $>10$; СРБ>20 мг/л), все больные получали МТ в сравнимых дозировках в качестве БПВП, около $30 \%$ пациентов во всех группах получали глюкокортикоиды (ГК).

При включении в исследование пациенты различных групп статистически значимо не отличались между собой по всем показателям PROs (табл. 2)

Терапия препаратом ОК3 64 мг к2н и к4н привела к значительному улучшению показателей PROs по сравнению с плацебо через 12 недель, и это улучшение сохранялось до 24-й недели терапии (рис. 1-6). В частности, динамика среднего значения функциональной недостаточности отражена на графике: по сравнению с исходным уровнем значение индекса HAQ-DI уменьшилось через 12 и 24 недели в группе ОК3 64 мг к2н на $0,54 \pm 0,04$ и $0,55 \pm 0,05$ бал-

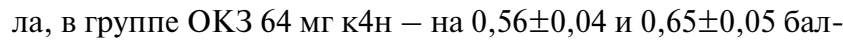

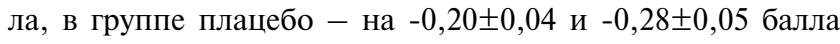
соответственно $(p<0,0001)$ (рис. 1$)$.

ООАЗП (PtGA) по ВАШ на фоне терапии OK3 64 мг к2н уменьшилась через 12 и 24 недели, соответственно, на $30,6 \pm 1,72$ и $32,1 \pm 1,92$ мм, в груп-

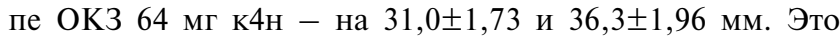
на $17,5 \pm 2,45$ и 17,9 $\pm 2,46$ мм больше по сравнению с показателями в группе плацебо на 12 -й неделе и на $12,7 \pm 2,73$ и 16,8土2,76 мм больше по сравнению с показателями в группе плацебо на 24-й неделе наблюдения для групп ОК3 64 мг к2н и ОК3 64 мг к4н соответственно (рис. 2).

Положительная динамика уменьшения выраженности боли по ВАШ была статистически значимо более существенной на фоне терапии ОКЗ: через 12 и 24 недели

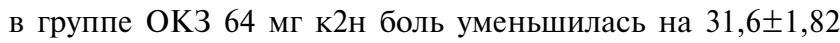
и $34,5 \pm 2,05$ мм, в группе ОК3 64 мг к4н - на $31,8 \pm 1,83$ 
Таблица 1. Общая характеристика пациентов РА при включении в исследование

\begin{tabular}{|c|c|c|c|}
\hline Показатели & ОК3 64 мг к2н (n=143) & ОКЗ 64 мг к4н $(n=142)$ & Плацебо ( $n=143)$ \\
\hline Возраст, годы & $52,0 \pm 11,8$ & $49,1 \pm 12,1$ & $52,7 \pm 11,3$ \\
\hline Пол, ж/м, $n(\%)$ & $116 / 27(81,1 / 18,9)$ & $118 / 24(83,1 / 16,9)$ & $120 / 23(83,9 / 16,1)$ \\
\hline Длительность РА, годы & $8,7[0,3 ; 36,3]$ & $7,3[0,3 ; 35,5]$ & $8,4[0,3 ; 37,0]$ \\
\hline ЧБС & $24,4 \pm 11,4$ & $22,2 \pm 10,2$ & $24,0 \pm 11,3$ \\
\hline ЧПС & $14,8 \pm 6,5$ & $14,5 \pm 6,7$ & $14,6 \pm 6,8$ \\
\hline DAS28-СРБ, баллы & $6,0 \pm 0,7$ & $5,9 \pm 0,7$ & $6,0 \pm 0,8$ \\
\hline СРБ, мг/л & $23,5 \pm 23,0$ & $22,7 \pm 22,7$ & $25,8 \pm 28,7$ \\
\hline Принимают ГК, $n$ (\%) & $52(36,4)$ & $50(35,2)$ & $41(28,7)$ \\
\hline Доза МТ, мг/нед. & $16,1 \pm 3,3$ & $16,3 \pm 3,4$ & $16,1 \pm 3,7$ \\
\hline
\end{tabular}

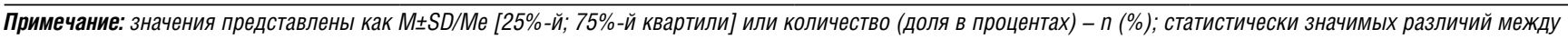
группами не обнаружено.

Таблица 2. Исходы, оцениваемые пациентом (PROS) при включении в исследование

\begin{tabular}{|c|c|c|c|}
\hline Исходы, оцениваемые пациентом (PROs) (M士SD) & 0К3 64 мг к2н ( $n=143)$ & ОК3 64 мг к4н (n=142) & Плацебо ( $n=143)$ \\
\hline HAQ-DI, баллы & $1,74 \pm 0,47$ & $1,64 \pm 0,50$ & $1,78 \pm 0,49$ \\
\hline ООАЗП (PtGА) ВАШ, Мм & $70,4 \pm 16,0$ & $68,5 \pm 14,5$ & $69,6 \pm 15,9$ \\
\hline Боль (ВАШ), мм & $70,2 \pm 16,3$ & $67,4 \pm 18,5$ & $68,3 \pm 17,6$ \\
\hline SF-36 (физический компонент), баллы & $31,7 \pm 6,29$ & $32,9 \pm 6,59$ & $31,8 \pm 6,51$ \\
\hline SF-36 (психический компонент), баллы & $42,9 \pm 11,3$ & $42,5 \pm 10,1$ & $41,4 \pm 8,34$ \\
\hline EQ-5D (ВАШ), мM & $39,2 \pm 19,6$ & $42,5 \pm 19,6$ & $39,1 \pm 20,6$ \\
\hline FACIT-F, балл & $26,7 \pm 9,06$ & $27,1 \pm 8,60$ & $26,7 \pm 8,39$ \\
\hline
\end{tabular}

Примечание: значения представлены как среднее для группы $M \pm S D$; статистически значимых различий между группами не обнаружено.

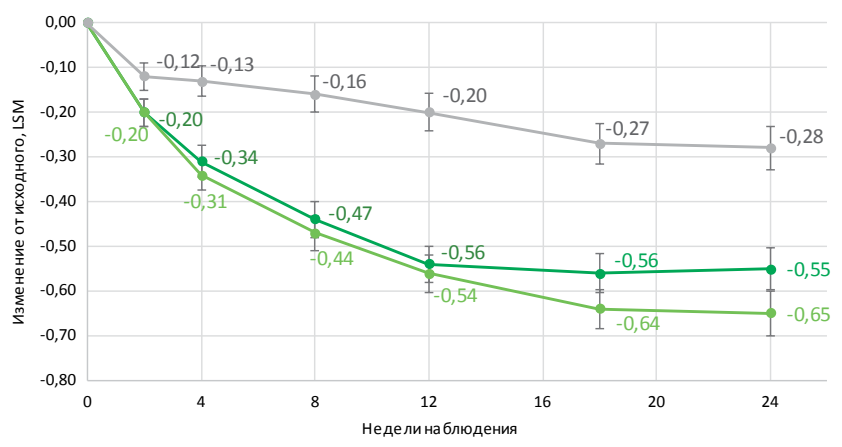

$\longrightarrow$-окз 64 мг к2н $\longrightarrow$ ОК364 мг к4н $\longrightarrow$ плацебо

Pис. 1. Динамика средних значений индекса HAQ-DI (баллы) до 24-й недели наблюдения

Примечание: LSM - среднее значение, рассчитанное по методу наименьших квадратов; к4н - каждые 4 недели, к2н - каждые 2 недели. Для каждой временной точки представлена стандартная ошибка среднего.

и $37,1 \pm 2,08$ мм соответственно. Это больше, чем в группе плацебо в эти же сроки, соответственно на $18,7 \pm 2,60$

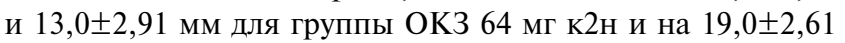

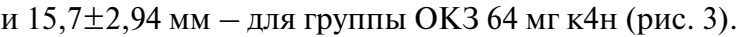

Терапия ОКЗ была более эффективной в отношении улучшения физического компонента шкалы SF-36: средние значения (LSM) данной подшкалы увеличились через 12 и 24 недели в группе ОК3 64 мг к2н соответственно на $6,72 \pm 0,57$ и 7,84 $\pm 0,67$ балла, что на $4,53 \pm 0,81$

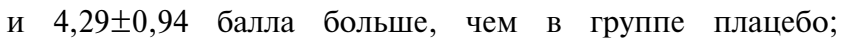
в группе ОК3 64 мг к4н - соответственно на 6,03 $\pm 0,57$ и $8,73 \pm 0,68$ балла, что на $3,84 \pm 0,82$ и $5,19 \pm 0,96$ балла больше, чем в группе плацебо. Психический компонент шкалы SF-36 также статистически значимо

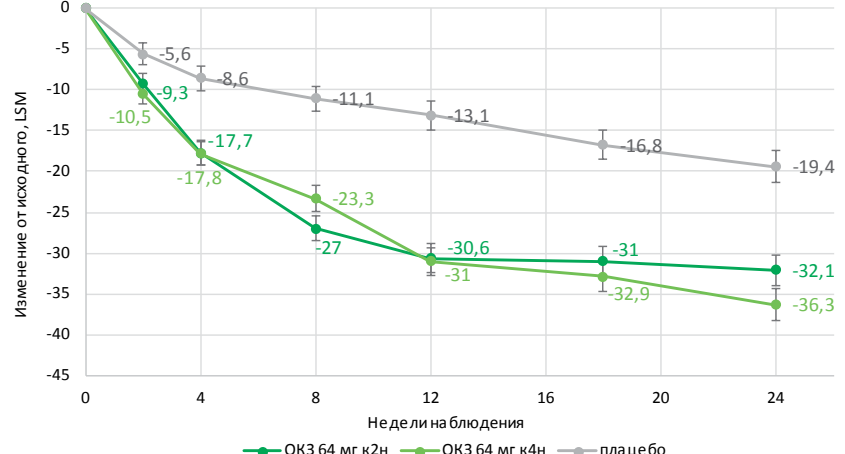

Рис. 2. Динамика средних значений ООАЗП по ВАШ (мм) до 24-й недели наблюдения

Примечание: LSM - среднее значение, рассчитанное по методу наименьших квадратов; к4н - каждые 4 недели, к2н - каждые 2 недели. Для каждой временной точки представлена стандартная ошибка среднего.

улучшился на фоне терапии ОКЗ через 12 и 24 недели: в группе ОК3 64 мг к2н - соответственно на $6,48 \pm 0,73$ и $6,22 \pm 0,79$ балла, что на $3,01 \pm 1,04$ и $3,72 \pm 1,11$ балла больше, чем в группе плацебо; в группе ОК3 64 мг к4н - соответственно на $7,04 \pm 0,73$ и $8,86 \pm 0,80$ балла, что на $3,57 \pm 1,04$ и $6,37 \pm 1,14$ балла больше, чем в группе плацебо (рис. 4).

Качество жизни (EQ-5D) на фоне терапии ОK3 статистически значимо улучшилось через 12 и 24 неде-

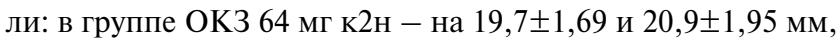

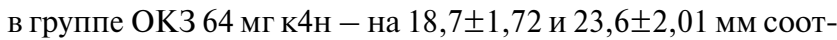
ветственно. Это больше, чем в группе плацебо в те же сро-

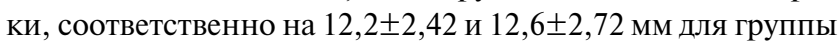

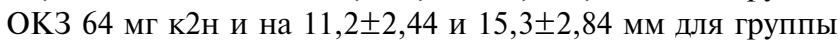
ОК3 64 мг к4н (рис. 5). 


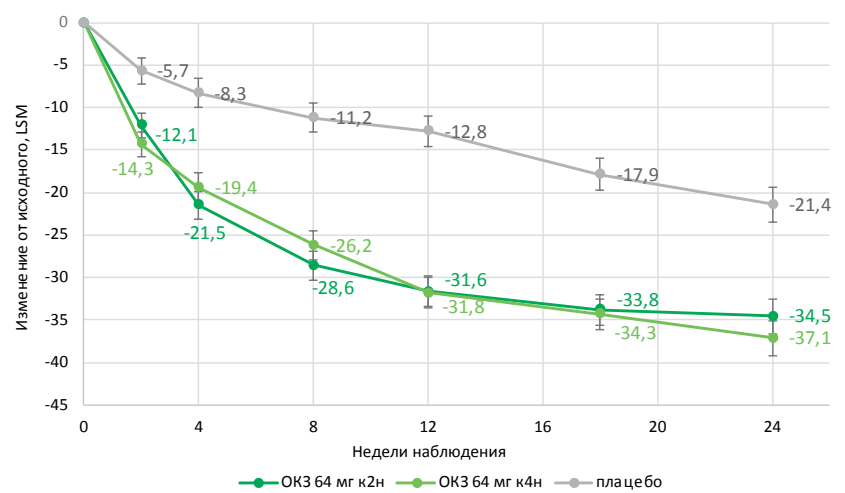

Рис. 3. Динамика средних значений выраженности боли по ВАШ (мм) до 24-й недели наблюдения

Примечание: LSM - среднее значение, рассчитанное по методу наименьших квадратов; к4н - каждые 4 недели, к2н - каждые 2 недели. Для каждой временной точки представлена стандартная ошибка среднего.
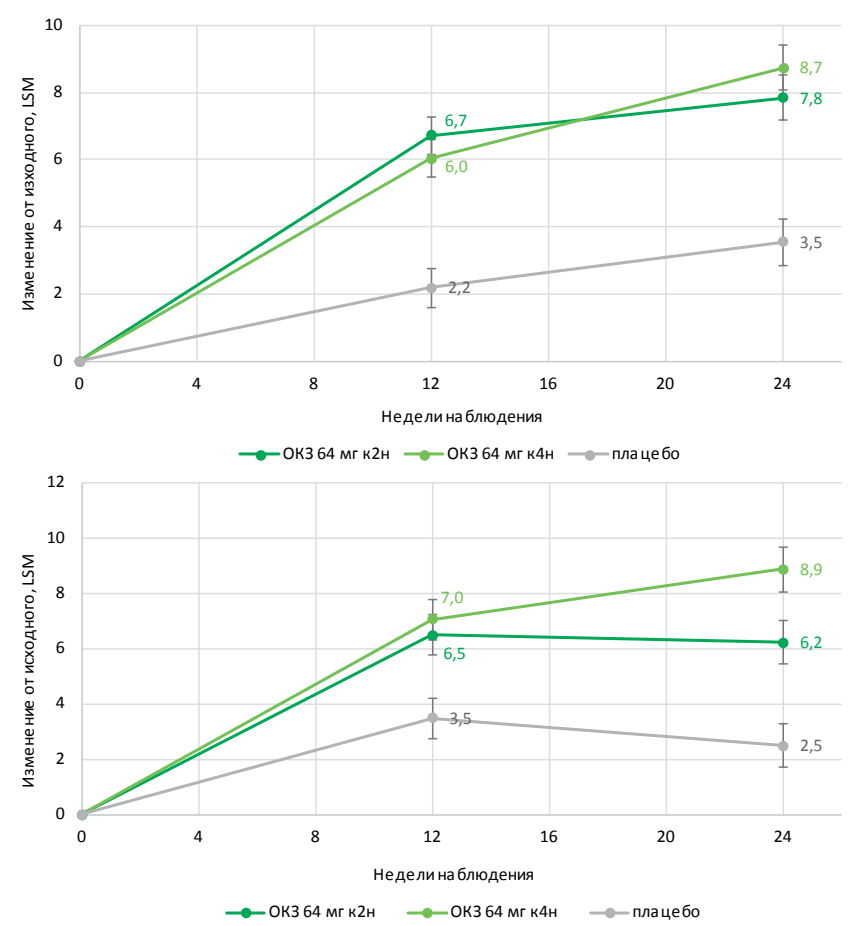

Pис. 4. Динамика средних значений фризического и психического компонентов шкалы качества жизни SF-36 (баллы) до 24-й недели наблюдения

Примечание: LSM - среднее значение, рассчитанное по методу наименьших квадратов; к4н - каждые 4 недели, к2н - каждые 2 недели. Для каждой временной точки представлена стандартная ошибка среднего.

Выраженность усталости (FACIT-F) через 12 и 24 недели улучшилась в группе ОК3 64 мг к2н, соответственно на $8,2 \pm 0,67$ и $8,5 \pm 0,80$ балла, что на $4,6 \pm 0,97$ и $4,8 \pm 1,12$ балла больше, чем в группе плацебо. В группе ОК3 64 мг к4н данный показатель улучшился соответственно на 8,7 $\pm 0,68$ и $10,6 \pm 0,81$ балла, что на $5,1 \pm 0,97$ и $6,9 \pm 1,14$ балла больше, чем в группе плацебо (рис. 6).

Число пациентов, достигших и превысивших минимальные клинически значимые различия (MCID) через 12 и 24 недели наблюдения, было статистически значимо больше в обеих группах ОКЗ по сравнению с группой плацебо для ООАЗП (PtGA), боли по ВАШ, HAQ-DI, FACIT-F. Число больных, достигших или превысивших MCID по физическому компоненту шкалы

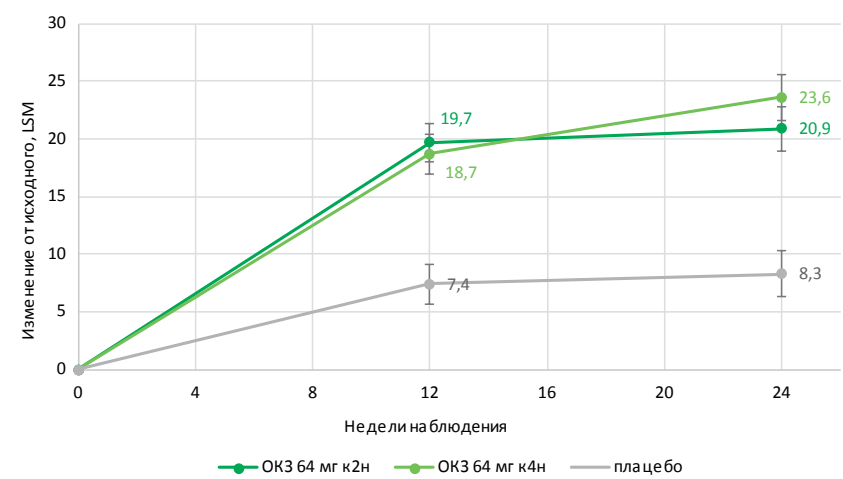

Pис. 5. Динамика средних значений шкалы качества жизни EQ-5D до 24-й недели наблюдения

Примечание: LSM - среднее значение, рассчитанное по методу наименьших квадратов; к4н - каждые 4 недели, к2н - каждые 2 недели. Для каждой временной точки представлена стандартная ошибка среднего.

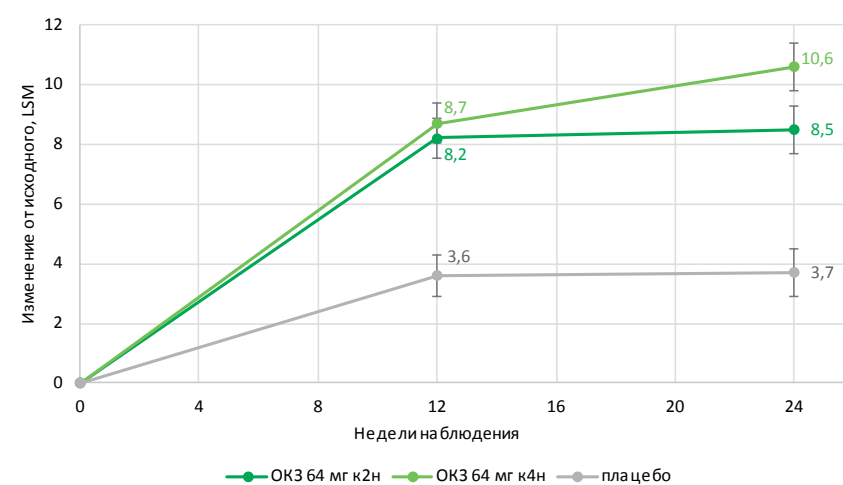

Pис. 6. Динамика средних значений шкалы усталости FACIT-F (баллы) до 24-й недели наблюдения

Примечание: LSM - среднее значение, рассчитанное по методу наименьших квадратов; к4н - каждые 4 недели, к2н - каждые 2 недели. Для каждой временной точки представлена стандартная ошибка среднего.

качества жизни SF-36 на 12-й неделе, было статистически значимо больше в обеих группах ОК3, а на 24-й неделе - только в группе ОК3 64 мг к4н по сравнению с группой плацебо. Психический компонент SF-36 улучшился у статистически значимо большего процента пациентов из группы ОК3 64 мг к4н по сравнению с группой плацебо на 12-й и 24-й неделях, в то время как группа ОК3 64 мг к2н статистически значимо не отличалась от группы плацебо по улучшению психического компонента SF-36 (рис. 7, 8).

\section{Обсуждение}

РА относится к хроническим прогрессирующим заболеваниям, которые негативно влияют на качество жизни пациентов, прежде всего за счет постоянной боли, усталости, тревожно-депрессивной симптоматики и функциональных ограничений [4, 31, 32]. Помимо снижения воспалительной активности и прогрессирования деструкции суставов, одной из основных задач терапии пациентов с РА является улучшение качества их жизни. Так как нередко оценка исходов болезни, по мнению врача и пациента, различается, а влияние РА на повседневную жизнь может оценить только сам пациент, включение показателей PROs для оценки эффективности терапии РА представляется обоснованным [9].

Научно-практическая ревматология. 2021;59(1):62-69 


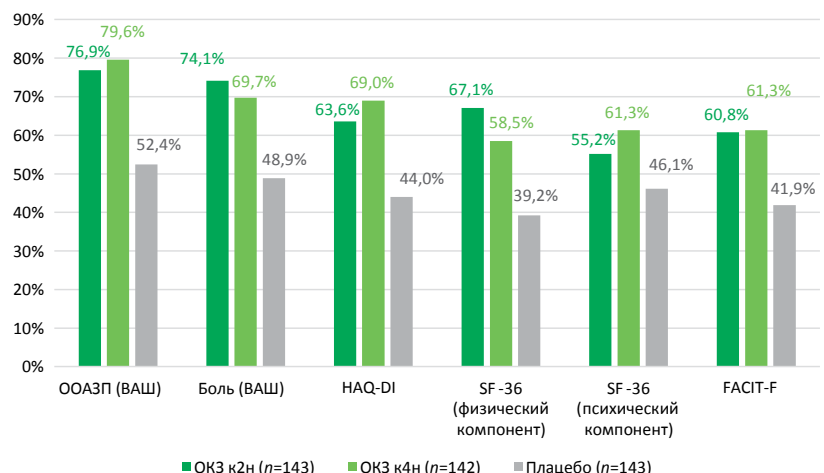

Рис. 7. Доля пациентов, достигших и превысивших минимальную клинически значимую разницу (MCID) по PROs к 12-й неделе наблюдения

Примечание: для разности групп ОКЗ с группой плацебо были рассчитаны точечные оценки и 97,5\% ДИ методом Ньюкомба - Уилсона. На 12-й неделе наблюдения 97,5\% ДИ не включают ноль для всех показателей, кроме SF-36 (психический компонент) для группы ОКЗ 64 мг к2н. Разность группы ОКЗ 64 мг К4н с группой плацебо (97,5\% ДИ): ООАЗП - 27,13\% (16,67-38,52): HAQ-DI - 24,96\% (13,95-36,92); боль по ВАШ - 20,77\% (9,66-32,66); FACIT-F 19,31\% (7,96-31,41); SF-36 (фиизический компонент) - 19,29\% (7,94-31,39); SF-36 (психический компонент) - 15,11\% (3,62-27,19); Разность группь ОКЗ 64 мг к2н с группой плацебо (97,5\% ДИ): боль по ВАШ - 25,17 (14,3836,89); ООАЗП - 24,48\% (13,82-35,99); HAQ-DI - 19,58\% (8,29-31,63); FACIT-F - 18,88\% (7,53-30,96); SF-36 (фиизический компонент) - 27,97\% (17,06-39,96); SF-36 (психический компонент) - 9,09\% $(-2,60-21,14)$.

Применение ингибиторов ИЛ-6 в терапии больных РА ассоциируется прежде всего с высокой эффективностью в отношении снижения клинико-лабораторных симптомов воспаления, хорошим профилем безопасности, а также с возможностью монотерапии $[11,33]$. Кроме того, поскольку ИЛ-6 принимает участие в развитии нейроэндокринных и нейрофизиологических нарушений при РА, его ингибиция может быть особенно эффективной в отношении широкого спектра клинических симптомов, характеризующих качество жизни, оцениваемое пациентами [34-36]. Данные, полученные в РКИ CREDO 1, свидетельствуют о выраженном положительном эффекте нового ингибитора ИЛ-6 - ОКЗ в дозе 64 мг подкожно каждые 2 и каждые 4 недели - в отношении всех исходов, оцениваемых пациентом - ООАЗП (PtGA), боли, усталости, функциональных возможностей, физической и психической составляющих качества жизни, что совпадает с полученными ранее данными, касающимися ингибиторов ИЛ-6Р, таких как тоцилизумаб (ТЦ3) [37, 38] и сарилумаб [39-41].

Несмотря на положительное влияние ингибиторов ИЛ-6 на психический компонент шкал качества жизни, а также на то, что депрессия, по данным многочисленных исследований, является самым частым коморбидным расстройством больных РА и оказывает существенное негативное воздействие на оценку эффективности проводимой терапии [42], прежде всего за счет негативного влияния на PROs [43], до настоящего времени ни в одном из исследований, посвященных оценке эффективности этих препаратов, в том числе ОК3, не проводилась оценка влияния препаратов на выраженность депрессии. В то же время предварительные результаты свидетельствуют о положительных эффектах ингибиторов ИЛ-6 в отношении большого депрессивного расстройства (БДР) [44], биполярного аффективного расстройства [45] и шизофрении [46]. В настоящее время инициированы РКИ фазы II, изучающие эффективность и переносимость

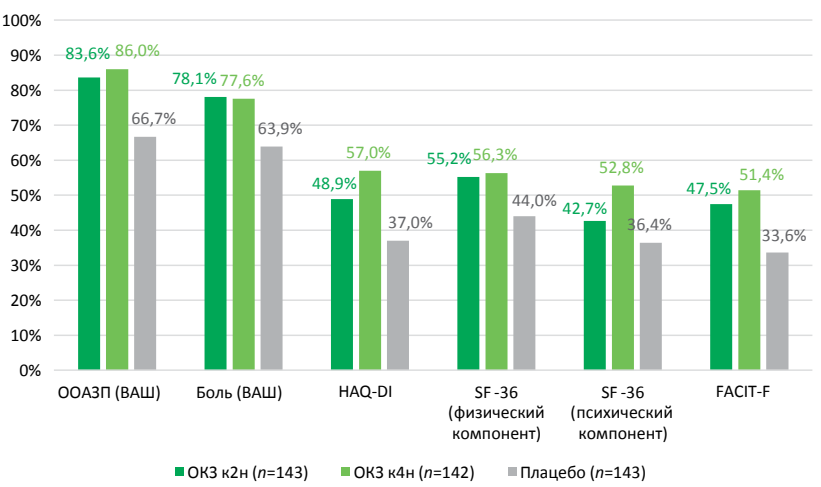

Рис. 8. Доля пациентов, достигших и превысивших минимальную клинически значимую разницу (MCID) по PROs к 24-й неделе наблюдения

Примечание: для разности групп ОКЗ с группой плацебо были рассчитаны точечные оценки и 97,5\% ДИ методом Ньюкомба - Уилсона. На 24-й неделе наблюдения 97,5\% ДИ не включают ноль для всех показателей, кроме SF-36 (психический и физический компоненты) для группы ОКЗ 64 мг к2н. Разность группы ОКЗ 64 мг К4н с группой плацебо (97,5\% ДИ): HAQ-DI - 19,98\% (8,6732,06); ООАЗП - 19,35\% (9,56-29,62); FACIT-F-17,84\% (6,54-29,81); боль по ВАШ - 13,73\% (3,14-24,76); SF-36 (психический компонент) - 16,45\% (5,0428,49); SF-36 (физический компонент) - 12,28\% (0,66-24,37). Разность группы ОКЗ 64 мг К2н с группой плацебо (97,5\% ДИ): ООАЗП - 16,89\% (6,9027,32); боль по ВАШ - 14,19\% (3,68-25,13); FACIT-F - 13,99\% (2,63-25,86); HAQ-DI-11,89\% (0,38-23,85); SF-36 (физический компонент) - 11,19\% $(-0,44-23,25) ;$ SF-36 (психический компонент) - 6,29\% $(-5,24-18,06)$.

сирукумаба (NCT02473289), ТЦ3 (NCT02660528) как дополнительных к антидепрессантам препаратов для лечения БДР у взрослых, силтуксимаба (NCT02796859) и ТЦЗ (NCT02034474 и NCT02874573) для лечения шизофрении [47]. Представляется обоснованным и перспективным в будущем инициировать исследования по влиянию ОК3 на выраженность депрессии у больных РА.

Таким образом, РКИ CREDO 1 продемонстрировало выраженную эффективность нового ингибитора ИЛ-6 ОКЗ в отношении исходов, оцениваемых самим пациентом: боли, усталости, ООАЗП, функциональных возможностей, качества жизни (физического и психического компонентов шкалы SF-36), - по сравнению с плацебо. Полученные результаты исследования в отношении PROs были сравнимы с подобными, продемонстрированными для других ингибиторов ИЛ-6 - ТЦЗ, сарилумаба и сирукумаба.

\section{Дополнительная информация}

Конфликт интересов. Авторы декларируют отсутствие явных и потенциальных конфликтов интересов, связанных с публикацией настоящей статьи. Кузькина С.М. является научным советником компании «Р-Фарм».

\section{Прозрачность исследования}

Исследование проведено при спонсорской поддержке компании «Р-ФАРМ». Авторы несут полную ответственность за предоставление окончательной версии рукописи в печать. Представленная работа не была ранее опубликована в других изданиях.

Декларация о финансовых и других взаимоотношениях

Все авторы принимали участие в разработке концепции статьи и в написании рукописи. Окончательная версия рукописи была одобрена всеми авторами. Авторы не получали гонорар за статью. 


\section{Л И Т Е Р А У P A / REFERE N C E S}

1. Smolen JS, Aletaha D, McInnes IB. Rheumatoid arthritis. Lancet. 2016;388(10055):2023-2038. doi: 10.1016/S0140-6736(16)30173-8

2. Ревматология: Российские клинические рекомендации. Под ред. ЕЛ Насонова. М.: ГЭОТАР-Медиа;2017:464. [Nasonov EL (ed.). Rheumatology: Russian Clinical Guidelines. Moscow: GEOTAR-Media;2017:464 (In Russ.)].

3. Kingsley G, Scott IC, Scott DL. Quality of life and the outcome of established rheumatoid arthritis. Best Pract Res Clin Rheumatol. 2011;25(4):585-606. doi: 10.1016/j.berh.2011.10.003

4. An J, Nyarko E, Hamad MA. Prevalence of comorbidities and their associations with health-related quality of life and healthcare expenditures in patients with rheumatoid arthritis. Clin Rheumatol. 2019;38(10):2717-2726. doi: 10.1007/s10067-019-04613-2

5. Felson DT, Anderson JJ, Boers M, Bombardier C, Chernoff M, Fried B, et al. The American College of Rheumatology preliminary core set of disease activity measures for rheumatoid arthritis clinical trials. The Committee on Outcome Measures in Rheumatoid Arthritis Clinical Trials. Arthritis Rheum. 1993;36(6):729-740. doi: 10.1002/art.1780360601

6. Ramey DR, Raynauld JP, Fries JF. The health assessment questionnaire 1992: status and review. Arthritis Care Res. 1992;5(3):119129. doi: 10.1002/art.1790050303

7. Strand V, Singh JA. Improved health-related quality of life with effective disease-modifying antirheumatic drugs: evidence from randomized controlled trials. Am J Manag Care. 2008;14(4):234254.

8. Van Tuyl LH, Michaud K. Patient-reported outcomes in rheumatoid arthritis. Rheum Dis Clin North Am. 2016;42(2):219-237. doi: 10.1016/j.rdc.2016.01.010

9. Kirwan JR, Hewlett SE, Heiberg T, Hughes RA, Carr M, Hehir M, et al. Incorporating the patient perspective into outcome assessment in rheumatoid arthritis - progress at OMERACT 7. J Rheumatol. 2005;32:2250-2256.

10. Генно-инженерные биологические препараты в лечении ревматоидного артрита. Под ред. ЕЛ Насонова. М.: ИМАПРЕСС;2013. [Nasonov EL (ed.). Genetically engineered biological drugs in the treatment of rheumatoid arthritis. Moscow: IMAPRESS;2013 (In Russ.)].

11. Насонов ЕЛ, Лила АМ. Ингибиция интерлейкина 6 при иммуновоспалительных ревматических заболеваниях: достижения, перспективы и надежды. Научно-практическая ревматология. 2017;55(6):590-599. [Nasonov EL, Lila AM. Inhibition of interleukin 6 in immune inflammatory rheumatic diseases: achievements, prospects, and hopes. NauchcnoPracticheskaya Revmatologia = Rheumatology Science and Practice. 2017;55(6):590-599 (In Russ.)]. doi: 10.14412/19954484-2017-590-599

12. Narazaki M, Tanaka T, Kishimoto T. The role and therapeutic targeting of IL-6 in rheumatoid arthritis. Expert Rev Clin Immunol. 2017;13(6):535-551. doi: 10.1080/1744666X.2017.1295850

13. Avci AB, Feist E, Burmester GR. Targeting IL-6 or IL-6 receptor in rheumatoid arthritis: What's the difference? BioDrugs. 2018;32(6):531-546. doi: 10.1007/s40259-018-0320-3

14. Kretsos K, Jullion A, Zamacona M, Harari O, Shaw S, Boulanger B, et al. Model-based optimal design and execution of the first-inpatient trial of the anti-IL-6, olokizumab. $C P T$ Pharmacometrics Syst Pharmacol. 2014;3(6):e119. doi: 10.1038/ psp.2014.17

15. Kretsos K, Golor G, Jullion A, Hickling M, McCabe S, Shaw S, et al. Safety and pharmacokinetics of olokizumab, an anti-IL-6 monoclonal antibody, administered to healthy male volunteers: A randomized phase I study. Clin Pharmacol Drug Dev. 2014;3(5):388-395. doi: 10.1002/cpdd.121

16. Genovese MC, Fleischmann R, Furst D, Janssen N, Carter J, Dasgupta B, et al. Efficacy and safety of olokizumab in patients with rheumatoid arthritis with an inadequate response to TNF inhibitor therapy: outcomes of a randomised Phase IIb study. Ann Rheum Dis. 2014;73(9):1607-1615. doi: 10.1136/annrheumdis-2013-204760
17. Takeuchi T, Tanaka Y, Yamanaka H, Amano K, Nagamine R, Park W, et al. Efficacy and safety of olokizumab in Asian patients with moderate-to-severe rheumatoid arthritis, previously exposed to anti-TNF therapy: Results from a randomized phase II trial. Mod Rheumatol. 2016;26(1):15-23. doi: 10.3109/14397595.2015.1074648

18. Nasonov E, Fatenejad S, Korneva E, Krechikova D, Maslyansky A, Plaksina T, et al. Safety and efficacy of olokizumab in a phase III trial of patients with moderately to severely active rheumatoid arthritis inadequately controlled by methotrexate CREDO1 study [abstract]. Arthritis Rheumatol. 2019;71(suppl 10). URL: https://acrabstracts.org/abstract/safety-and-efficacy-of-olokizumab-in-a-phase-iii-trial-of-patients-with-moderately-to-severely-active-rheumatoid-arthritis-inadequately-controlled-by-methotrexate-credo1-study (accessed July 7, 2020).

19. Nasonov E, Stoilov R, Tyabut T on behalf of Saeed Fatenejad (United States of America), Krechikova D., Korneva E., Maslyansky A., et al. OP0021 olokizumab, monoclonal antibody against IL6, in patients with moderately to severely active rheumatoid arthritis inadequately controlled by methotrexate: Efficacy and safety results of phase III CREDO-1 study. Annals of the Rheumatic Diseases. 2020;79:16-17. doi: 10.1136/annrheumdis-2020-eular.1688

20. Anderson JK, Zimmerman L, Caplan L, Michaud K. Measures of rheumatoid arthritis disease activity: Patient (PtGA) and Provider (PrGA) Global Assessment of Disease Activity, Disease Activity Score (DAS) and Disease Activity Score with 28-Joint Counts (DAS28), Simplified Disease Activity Index (SDAI), Clinical Disease Activity Index (CDAI), Patient Activity Score (PAS) and Patient Activity Score-II (PASII), Routine Assessment of Patient Index Data (RAPID), Rheumatoid Arthritis Disease Activity Index (RADAI) and Rheumatoid Arthritis Disease Activity Index-5 (RADAI-5), Chronic Arthritis Systemic Index (CASI), PatientBased Disease Activity Score With ESR (PDAS1) and PatientBased Disease Activity Score without ESR (PDAS2), and Mean Overall Index for Rheumatoid Arthritis (MOI-RA). Arthritis Care Res (Hoboken). 2011;63 Suppl 11:S14-36. doi: 10.1002/acr.20621

21. Cella D, Yount S, Sorensen M, Chartash E, Sengupta N, Grober J. Validation of the Functional Assessment of Chronic Illness Therapy Fatigue Scale relative to other instrumentation in patients with rheumatoid arthritis. J Rheumatol. 2005;32(5):811-819.

22. Hurst NP, Jobanputra P, Hunter M, Lambert M, Lochhead A, Brown H. Validity of Euroqol - a generic health status instrument - in patients with rheumatoid arthritis. Economic and Health Outcomes Research Group. Br J Rheumatol. 1994;33(7):655-662. doi: 10.1093/rheumatology/33.7.655

23. Ware JE, Kosinski M, Keller SK: SF-36 physical and mental health summary scales: A user's manual. Boston: New England Medical Center, The Health Institute;1994.

24. Fries JF, Spitz P, Kraines RG, Holman HR. Measurement of patient outcome in arthritis. Arthritis Rheum. 1980;23:137-145.

25. Prevoo ML, van't Hof MA, Kuper HH, van Leeuwen MA, van de Putte LB, van Riel PL. Modified disease activity scores that include twenty-eight-joint counts: development and validation in a prospective longitudinal study of patients with rheumatoid arthritis. Arthritis Rheum. 1995;38:44-48.

26. Aletaha D, Smolen J. The Simplified Disease Activity Index (SDAI) and the Clinical Disease Activity Index (CDAI): A review of their usefulness and validity in rheumatoid arthritis. Clin Exp Rheumatol. 2005;23(5 Suppl 39):S100-108.

27. Strand V, Boers M, Idzerda L, Kirwan JR, Kvien TK, Tugwell PS, et al. It's good to feel better but it's better to feel good and even better to feel good as soon as possible for as long as possible. Response criteria and the importance of change at OMERACT 10. J Rheumatol. 2011;38:1720-1727.

28. Амирджанова ВН, Горячев ДВ, Коршунов НИ, Ребров АП, Сороцкая В.Н. Популяционные показатели качества жизни по опроснику SF-36 (результаты многоцентрового исследования качества жизни «МИРАЖ»). Научно-практическая ревматология. 2008;46(1):36-48. [Amirdjanova VN, Goryachev DV, Korshunov NI, Rebrov AP, Sorotskaya VN. SF-36 questionnaire 
population quality of life indices. Nauchcno-Practicheskaya Revmatologia $=$ Rheumatology Science and Practice. 2008;46(1):3648 (In Russ.)]. doi: 10.14412/1995-4484-2008-852

29. Wells GA, Tugwell P, Kraag GR, Baker PR, Groh J, Redelmeier DA. Minimum important difference between patients with rheumatoid arthritis: the patient's perspective. J Rheumatol. 1993;20:557-560.

30. Lubeck DP. Patient-reported outcomes and their role in the assessment of rheumatoid arthritis. Pharmacoeconomics. 2004;22:27-38.

31. Matcham F, Scott IC, Rayner L, Hotopf M, Kingsley GH, Norton $\mathrm{S}$, et al. The impact of rheumatoid arthritis on quality-oflife assessed using the SF-36: a systematic review and meta-analysis. Semin Arthritis Rheum. 2014;44(2):123-130. doi: 10.1016/j. semarthrit.2014.05.001

32. Heaney A, Stepanous J, Rouse M, McKenna SP. A review of the psychometric properties and use of the Rheumatoid Arthritis Quality of Life Questionnaire (RAQoL) in clinical research. Curr Rheumatol Rev. 2017;13(3):197-205. doi: 10.2174/157339631366617 0615092324

33. Choy EH, De Benedetti F, Takeuchi T, Hashizume M, John MR, Kishimoto T. Translating IL-6 biology into effective treatments. Nat Rev Rheumatol. 2020;16(6):335-345. doi: 10.1038/s41584-020-0419-z

34. Choy EHS, Calabrese LH. Neuroendocrine and neurophysiological effects of interleukin 6 in rheumatoid arthritis. Rheumatology (Oxford). 2018;57(11):1885-1895. doi: 10.1093/rheumatology/kex391

35. Лисицына ТА, Вельтищев ДЮ, Лила АМ, Насонов ЕЛ. Интерлейкин 6 как патогенетический фактор, опосредующий формирование клинических проявлений, и мишень для терапии ревматических заболеваний и депрессивных расстройств. Научно-практическая ревматология. 2019;57(3):318-327. [Lisitsyna TA, Veltishchev DYu, Lila AM, Nasonov EL. Interleukin 6 as a pathogenic factor mediating clinical manifestations and a therapeutic target for rheumatic diseases and depressive disorders. Nauchcno-Practicheskaya Revmatologia $=$ Rheumatology Science and Practice. 2019;57(3):318-327 (In Russ.)]. doi: 10.14412/1995-4484-2019-318-327

36. Matcham F, Galloway J, Hotopf M, Roberts E, Scott IC, Steer S, et al. The impact of targeted rheumatoid arthritis pharmacologic treatment on mental health: A systematic review and network meta-analysis. Arthritis Rheumatol. 2018;70(9):1377-1391. doi: 10.1002/art.40565

37. Strand V, Michalska M, Birchwood C, Pei J, Tuckwell K, Finch R, et al. Impact of tocilizumab administered intravenously or subcutaneously on patient-reported quality-of life outcomes in patients with rheumatoid arthritis. RMD Open. 2018;4:e00602. doi: 10.1136/ rmdopen-2017-000602
38. Sebba A, Han J, Mohan S. Pain and other patient-reported outcomes in patients with rheumatoid arthritis who did or did not achieve treatment response based on improvement in swollen joints in tocilizumab clinical trials [abstract]. Arthritis Rheumatol. 2020; 72(suppl 10). URL: https://acrabstracts.org/abstract/pain-andother-patient-reported-outcomes-in-patients-with-rheumatoidarthritis-who-did-or-did-not-achieve-treatment-response-basedon-improvement-in-swollen-joints-in-tocilizumab-clinical-trials/ (accessed July 7, 2020).

39. Crotti C, Biggioggero M, Becciolini A, Favalli EG. Sarilumab: patient-reported outcomes in rheumatoid arthritis. Patient Relat Outcome Meas. 2018;9:275-284. doi: 10.2147/PROM.S147286

40. Насонов ЕЛ, Лила АМ. Эффективность и безопасность сарилумаба (полностью человеческие моноклональные антитела к рецептору интерлейкина 6) при ревматоидном артрите: новые данные. Научно-практическая ревматология. 2019;57(5):564-577 [Nasonov EL, Lila AM. The efficacy and safety of sarilumab, fully human monoclonal antibodies against interleukin 6 receptor, in rheumatoid arthritis: new evidence. Nauchcno-Practicheskaya Revmatologia $=$ Rheumatology Science and Practice. 2019;57(5):564-577 (In Russ.)]. doi: 10.14412/19954484-2019-564-57

41. Atzeni F, Nucera V, Masala IF, Sarzi-Puttini P, Bonitta G. Il-6 involvement in pain, fatigue and mood disorders in rheumatoid arthritis and the effects of Il-6 inhibitor sarilumab. Pharmacol Res. 2019;149:104402. doi: 10.1016/j.phrs.2019.104402

42. Dougados M, Soubrier M, Antunez A, Balint P, Balsa A, Buch $\mathrm{MH}$, et al. Prevalence of comorbidities in rheumatoid arthritis and evaluation of their monitoring: results of an international, cross-sectional study (COMORA). Ann Rheum Dis. 2014;73(1):6268. doi: 10.1136/annrheumdis-2013-204223

43. Nerurkar L, Siebert S, McInnes IB, Cavanagh J. Rheumatoid arthritis and depression: an inflammatory perspective. Lancet Psychiatry. 2019;6(2):164-173. doi: 10.1016/S2215-0366(18)30255-4

44. Zhou AJ, Lee Y, Salvadore G, Hsu B, Fonseka TM, Kennedy SH, et al. Sirukumab: A potential treatment for mood disorders? Adv Ther. 2017;34:78-90. doi: 10.1007/s12325-016-0455-x

45. Brietzke E, Scheinberg M, Lafer B. Therapeutic potential of interleukin-6 antagonism in bipolar disorder. Medical Hypotheses. 2011;76(1):21-23. doi: 10.1016/j.mehy.2010.08.021

46. Miller BJ, Dias JK, Lemos HP, Buckley PF. An open-label, pilot trial of adjunctive tocilizumab in schizophrenia. J Clin Psychiatry. 2016;77:275-276. doi: 10.4088/JCP.15109920

47. Miller BJ, Buckley PF. Monoclonal antibody immunotherapy in psychiatric disorders. Lancet Psychiatry. 2017;4(1):13-15. doi: 10.1016/S2215-0366(16)30366-2
Насонов Е.Л. ORCID: https://orcid.org/0000-0002-1598-8360

Лисицына T.A. ORCID: https://orcid.org/0000-0001-9437-406X

Зонова E.B. ORCID: https://orcid.org/0000-0002-0228-9085

Кузькина C.M. ORCID: https://orcid.org/0000-0002-2230-5847 